\title{
Autonomous Mobile Learning Model of Cloud Education Based on Intelligent Algorithm of Wireless Network Communication
}

\author{
Lin Chen ${ }^{1}$ and Weiping Zhu $\mathbb{D}^{2}$ \\ ${ }^{1}$ School of Education Science, Chongqing Normal University, Chongqing, 401331 Chongqing, China \\ ${ }^{2}$ School of Humanities and Education, Nanchang Institute of Technology, Nanchang, 330044 Jiangxi, China \\ Correspondence should be addressed to Weiping Zhu; zwp@nut.edu.cn
}

Received 5 August 2021; Accepted 4 September 2021; Published 13 October 2021

Academic Editor: Zhihan Lv

Copyright ( 2021 Lin Chen and Weiping Zhu. This is an open access article distributed under the Creative Commons Attribution License, which permits unrestricted use, distribution, and reproduction in any medium, provided the original work is properly cited.

\begin{abstract}
The rapid development of a new generation of information technology and its widespread application in colleges and universities have promoted profound changes in campus informatization. On the one hand, wireless campus networks as the main infrastructure of digital campuses are gradually popularized in domestic colleges and universities, expanding the campus information network coverage. This article is aimed at studying the addition of intelligent algorithms to wireless network communications to optimize and build autonomous mobile learning for cloud education. In order for children to communicate with teachers face to face, no matter where they are, this article proposes how to reduce the energy loss in wireless communication and find the best intelligent algorithm to realize the cloud education mobile learning platform. Experimental results show that the transmission delay of the wireless communication system of this method is significantly shorter than that of the other two methods, close to 5\%, which speeds up the data transmission speed of the wireless communication system. It can be implemented under time-sensitive conditions and has high practical application value. The wireless network communication system of this method has a low packet loss rate and less wireless network data transmission errors, which is nearly $10 \%$ lower than the other two methods, thereby improving the data transmission power of the wireless communication network.
\end{abstract}

\section{Introduction}

1.1. Background. The applications of mobile learning and cloud education have been applied to the education of major foreign universities. MU Elementary School, using Simon's cloud service, can obtain students' computer information through Weibo and provide students with services to communicate and complete teaching tasks. Through the implementation of the "Classroom 2000" (c2k) project in Northern Ireland, more than 350,000 teachers and students in 1,200 primary schools can access the first "cloud" in Europe through wifi network services. Student education provides schools with high-quality online resources; teachers and student education can access and download various resources through mobile terminals and computers. In the context of cloud computing technology, a "cloud" supercomputer group has emerged, and service providers provide services to users based on this group; it is a safe and fast information storage method that allows users to quickly process information in a secure network environment. The application of mobile learning technology in the field of education and learning has given birth to a new learning method under the background of cloud computing-mobile learning. The American National Institute of Standards and Technology is a standardization organization in the United States. It defines cloud computing as a new Internet computing method. It builds a resource pool through the Internet to provide users with real-time updates, flexible expansion, and virtualized resources, mainly based on infrastructure as a service platform and as a service and software, and other service models are presented. Our country has been trying hard to try mobile learning applications. The first is applied research in various studies. In 2001, the Department of Higher Education of the Ministry of Education proposed a pilot project for the construction of a mobile learning platform and organized experts to carry out a 
scientific research project on "Mobile Education Theory and Practice." In 2002, some domestic schools began to build a wireless school campus network. Students from Hong Kong's Pui Ching Middle School can use their mobile phones to answer teacher's questions in real time in the classroom, improving the interactivity of learning. It is an inevitable product of cloud computing: one is the integration of cloud computing memory, which provides a platform for integrating massive learning resources, and two is cloud computing technology can effectively solve the problem of "information omission, insufficient storage, and limitations" of mobile learning media. Taiwan will also promote the development of mobile learning. With the continuous advancement of Internet technology, mobile learning can not only meet people's needs at any time. The demand for local autonomous learning and abundant educational resources will significantly reduce people's learning costs. With the rapid development of wireless network communication, we will find that our learning will become more convenient and effective.

1.2. Significance. Mobile learning activities in the cloud education platform can not only complete a series of actions in a sequence and make them a continuous flow of activities but also expand the field of activities and eliminate interference factors, thereby significantly improving learning efficiency. The development of information technology, especially the continuous development and progress of sensor technology and computer technology, and the continuous enhancement and improvement of various sensor functions and the continuous expansion of application fields make the working system more energy-efficient, more efficient, smaller in size, and lower in cost. Node location information and collected data will lose some practical use value of network balance. With the continuous advancement of sensor network technology, routing protocols based on location information and various monitoring applications can be better developed and improved.

1.3. Related Work. Cloud education is to use the concept of cloud computing to realize distributed management and joint application of teaching resources based on cloud computing technology to meet the needs of teachers for reforming teaching methods and realizing teaching resources. In personalized learning now, as long as you have a mobile device, you can realize online interactive learning and interactive learning. In Wang et al.'s research, in order to prevent sensor nodes from being easily captured, destroyed, or attacked in an open environment, which leads to the security and accuracy of data transmission, they proposed a trustbased perception model. The ant colony optimization algorithm for secure routing in wireless sensor networks can effectively identify and stay away from malicious nodes. In their research, although some problems have been solved, the research considerations are not very comprehensive, and there are still certain deficiencies. I hope that they can be better improved in future life [1]. Lin et al. deal with WSN security from the adversarial perspective and study low-cost and efficient algorithms to identify sensors in
WSN in the shortest time and at the lowest cost. It is recommended to convert the problem to a set covering problem and develop a greedy minimum cost node capture attack algorithm (MCA) to reduce the cost of the attack. Extensive simulations have been implemented to evaluate the performance of MCA and compare it with several related scenarios. The results showed that MCA reduced the cost of destroying WSN by $20 \%$. Although the research has great practical value and broad prospects, there are technical barriers and great challenges [2]. Tang et al. improved the Low-Energy Adaptive Clustering Hierarchy (LEACH) protocol, making it suitable for energy harvesting wireless sensor networks. However, there are still some problems in the experimental part of the study, and it does not take into account that there is a certain loss of energy [3]. Muhammad proposed a novel intelligent positioning algorithm that uses variable distance beacon signals generated by changing the transmission power of beacon nodes. The algorithm does not use any additional hardware resources for ranging and estimates the location by passively listening to beacon signals using only radio connections [4]. After summarizing its characteristics, it analyzes the possibility of combining it with mobile education, proposes a new model of mobile education based on cloud computing, and conducts exploratory research on the development of mobile education. Although the research is very forward-looking, there is still a problem of insufficient technology [4]. Sengottuvelan and Prasath pointed out that a wireless sensor network (WSN) consists of a large number of independent nodes with sensors, wireless interfaces for communication, and limited processing and energy resources [5]. Research is very practical, but the framework constructed in this way requires a lot of cost [5]. Alam and Saiyeda propose a solution that combines cloud technology and learning to obtain an optimized learning system that will help students conduct self-assessment and will use the cloud to provide their services. However, the experimental and analytical aspects are relatively simple and not detailed enough [6]. Kati and Khan pointed out that during the period of the new coronavirus, many countries have adopted online classes in order not to delay students from attending classes. Although online teaching is widely used, there are many challenges and security issues especially when using education clouds for educational dissemination. There is no guarantee that students are listening carefully when they are studying online, and there are also related mobile device delays and technical problems. These are huge problems. He can only increase the children's learning concentration by increasing the questions in the classroom. Although their research is very forward-looking, there is still no good experimental data to support [7].

1.4. Innovation. With the widespread construction of campus wireless networks and the increasing popularity of mobile terminals, mobile learning has become one of the most important learning methods for teachers and students, and they can learn anytime, anywhere. It does not meet the needs of space-time mobile phones, massive resource exchange, and differentiated terminal support, which creates obstacles for teachers to use. Based on the basic idea of cloud 
computing, cloud training transmits all the information from the client and realizes integrated management and resource planning. Based on the intelligent algorithm of wireless network communication, it realizes the purpose of autonomous mobile learning of cloud training model, which is more and more convenient and more suitable for us. The optimization of wireless networks and intelligent algorithms strengthens the communication between teachers and students, thereby improving children's learning efficiency. Through the combination of wireless network communication and intelligent algorithms, intelligent algorithms can reduce energy consumption during signal propagation. At the technical level, by optimizing the signal of wireless network communication equipment, by helping to reduce the energy consumption in the signal propagation process, ensuring that it can be transmitted farther and longer, a series of updates are made to this by using new materials and technologies developed. Wireless network communication realizes the communication in different spaces but will lose a lot of energy in the connection, but the emergence of intelligent algorithms perfectly fits wireless network communication. With the combination of wireless network communication and intelligent algorithms, cloud education autonomous mobile learning will produce brilliant sparks in the education industry.

\section{Methods and Related Concepts}

2.1. Wireless Network Communication. In modern times, with the rapid development of science and engineering technology, in the face of a large amount of data and information rushing to us, collection, transmission, monitoring, analysis, wireless sensors, and their composed wireless sensor networks have entered people's field of vision and gained rapid development and attention. The wireless sensor network integrates microsmart sensor technology, embedded computing technology, wireless communication technology, and distributed information processing technology, realtime sensing and collecting information about the environment or objects in the monitoring area. The development in the past two decades is mainly reflected in the continuous upgrading of hardware technology and the continuous maturity of various network-related technologies, and good research theoretical results and technical application results have been achieved. The function has attracted widespread attention. The following shows the research proportions of various directions in the number of publications in the database this year, and we can more intuitively observe the main research directions of wireless network communication, as shown in Figure 1.

From Figure 1(a), we can see that there was more research on new energy 15 years ago. Energy consumption is an important issue that we need to solve before. It is the basis for the development of other technologies, then network routing technology, and as can be seen from Figure 1(b), for key technical issues such as positioning, in continuous practice, more and more problems follow one after another. At present, people are increasingly aware of the importance of security and mobility issues. The intelligent era based on the Internet of Things has come, and the development of science and technology continues to spread to the field of life and production. As one of the key technologies, wireless sensor networks have received extensive attention. In response to the needs of the development of the times, some domestic scientific research institutes and universityrelated laboratories have carried out researches on the theory and application of wireless sensor networks. The laboratories of many universities and other units across the country have carried out research on wireless sensor networks from different angles, and the results of academic and practical applications have increased sharply, which has led to a climax of research [8]. In the monitoring area, a large number of sensor nodes are deployed to sense, collect, and monitor information in the designated area, and then, a certain route was used to transmit to the collection node, then through the Internet or satellite media, and through wired or wireless transmission, and finally, the host computer of the monitoring system arrived. User-issued tasks are fed back to each node in the opposite transmission mode. In the entire data transmission process, each sensor node must not only complete the task of collecting and forwarding data but also has the ability to connect to each other and communicate and locate. The architecture of wireless network communication is shown in Figure 2 [9].

2.2. Cloud Education Autonomous Mobile Learning Model. As an important support for mobile learning, cloud education has irreplaceable advantages over Moodle, Bio, and other network platforms. These unique advantages provide favorable conditions for the development of mobile learning and fully demonstrate the charm of mobile learning. Students' learning interest and strong learning interest make learning more active. The virtual learning environment provided by cloud education can separate teachers and students in time and space. Students can choose the time, place, and content of learning according to their needs. Based on the advantages of the cloud learning platform, we have built a stand-alone machine based on the cloud learning platform; the mobile learning model is shown in Figure 3 [10].

The process model of mobile learning activities based on the cloud education platform mainly includes three stages. The first is the initial analysis stage of mobile learning activities. This is a very important stage of mobile learning design. According to the analyzed learning content and student characteristics, determine whether the mobile learning mode is suitable for development. The second is the mobile learning activity design stage under cloud education. This stage includes the planning of mobile learning activity tasks, the establishment of its organizational form, and the design of activity processes and activity criteria and activity evaluation design. The third stage is the mobile learning environment design stage under the cloud education platform. This part of the work mainly includes related cloud hardware and cloud software ecological design and cloud humanistic ecological design.

Our model provides students with learning planning before, during, and after class. Before class, students can $\log$ in to the system to obtain the corresponding learning 


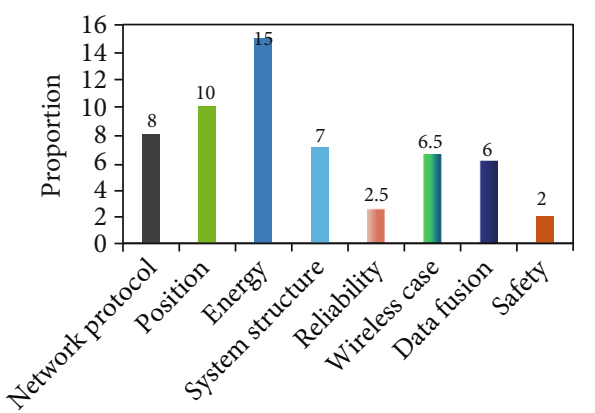

Research direction

(a)

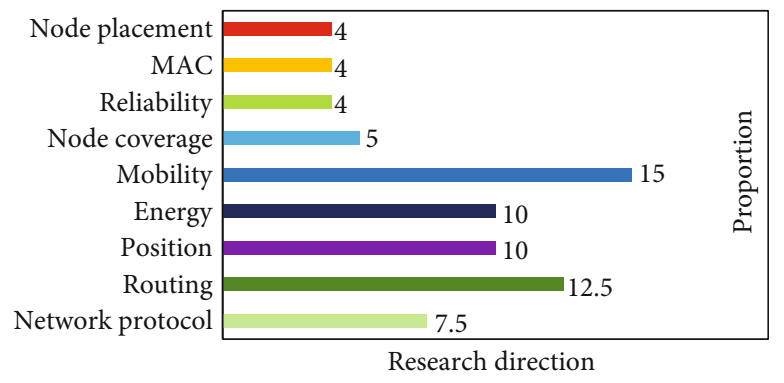

(b)

FIGURE 1: (a) Distribution map of wireless network research before 2015. (b) Distribution map of current wireless network research directions.

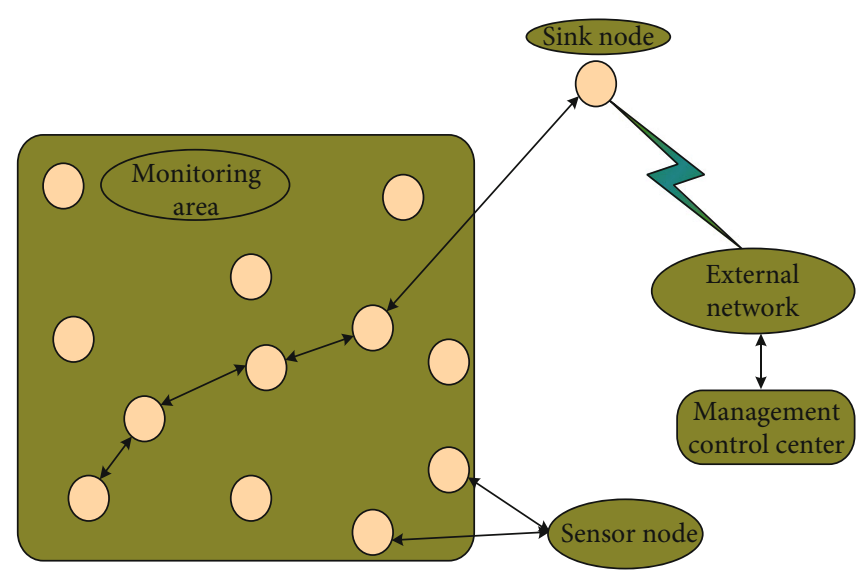

FIGURE 2: The architecture of wireless network communication.

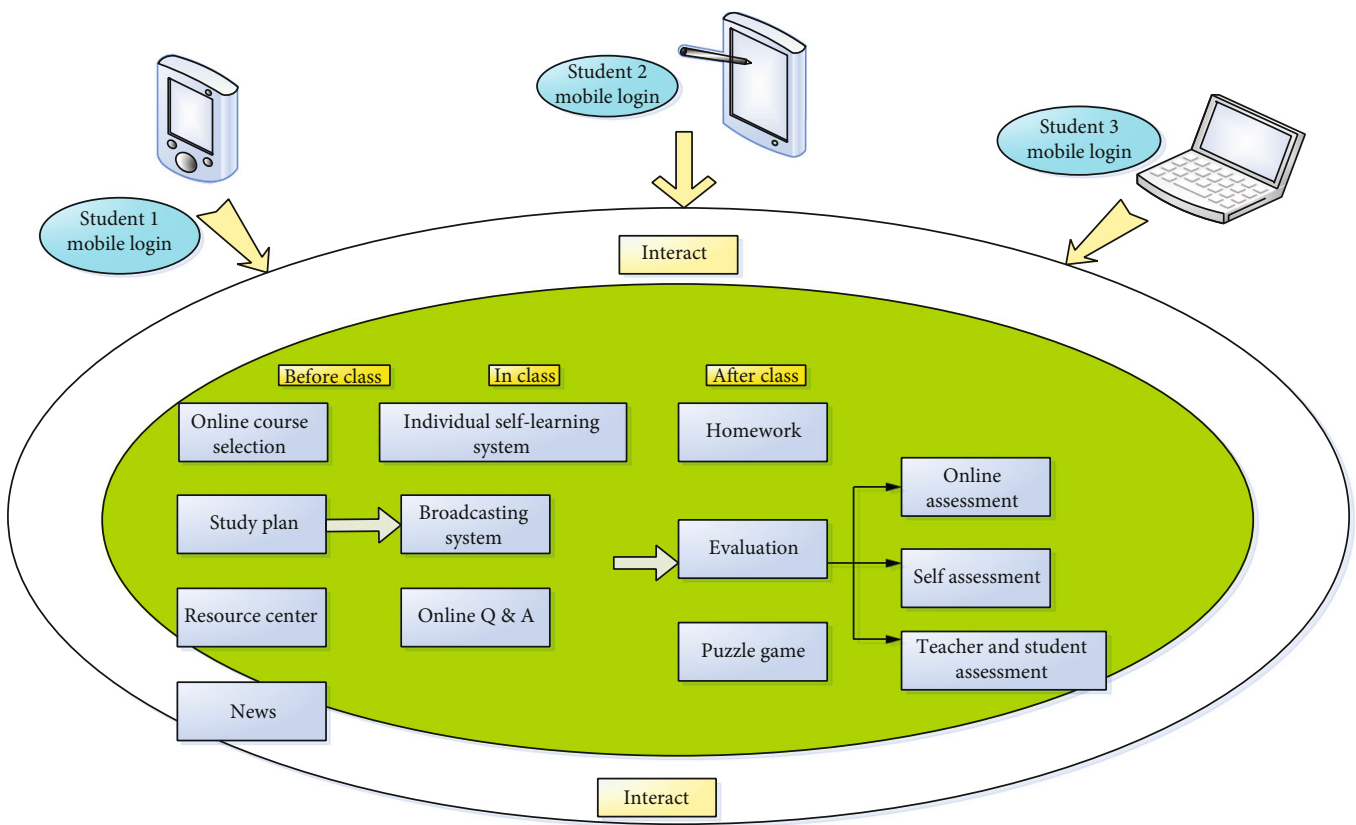

Figure 3: Autonomous mobile learning model for cloud education. 
materials and make sufficient preclass preparations. We can also choose according to our hobbies. In curriculum, interest is the child's first teacher and will also provide children with some news at home and abroad, so that children can learn about real-time news [11]. In the classroom, children do not need to carry heavy schoolbags to school. They can obtain the corresponding information based on mobile devices. When they encounter problems, they can ask teachers and classmates for help online at any time, which can help children online. For Q\&A, after class, children no longer have to worry about homework and forgetting the trouble. Our homework can be submitted anywhere. The premise is that there is a network. Some homework can be controlled by the teacher through the system. Students answer and fill in online, and this can provide timely feedback on the evaluation results to students and teachers, so that they can recognize their shortcomings and strengths in a timely manner and deepen their impression of the learning content. Educational classes provided by cloud education learning game of the game allow students to increase their knowledge and improve their learning skills in the game. The most important thing is that they can learn happily. Mobile learning must have three characteristics. The first important thing is to have digital teaching content, and the second is to use mobile media as learning tools, such as mobile phones, tablets, computers, and TVs, and teachers and students must be on a separated status; this is the criterion for mobile learning, as shown in Figure 4 [12].

For the application of future wireless network communication intelligent algorithms in the autonomous mobile learning model of cloud education, they will bring more intelligence and speed to our learning environment and consume less energy, and there will be less energy consumption between students and teachers. Communication is not limited to space. In this era of communication, no matter where we are, we can always pick up mobile devices to communicate with our teachers or students.

2.3. Intelligent Algorithm. The swarm intelligence algorithm is created by researchers based on the self-organizing behavior of social organisms, mathematically modeling swarm intelligence, and reconstructing this model with computers [13]. The collective foraging process of ant colonies and bird colonies reflects the limited ability of individuals [14]. However, complex tasks can be completed through cooperation and division of labor by grouping into groups [15]. So inspired by this, it provides new ideas for solving complex optimization problems. According to the behavior of ant colonies and bird colonies, the researchers, respectively, proposed an ant colony optimization algorithm and another representative particle swarm optimization algorithm through simulation analysis. The continuous in-depth development of the two has laid a good foundation for the future development of swarm intelligence, the basis of [16]. Applying it to cloud education, autonomous mobile learning will give people a completely new feeling, and the actual changes are shown in Figure 5 [17]. The advantage of intelligent algorithms and other algorithms is that they can help this cloud education learning model to perform a certain optimi- zation process, help to make the wireless network transmission process faster, and reduce certain network delays.

Under the cloud education mobile autonomous learning model of wireless network communication, we can not only learn some courses in school, if we do not hear clearly, we can also watch related videos according to the courses we have learned after returning home. There are also some video teachings and so on that we are interested in. We can come into contact with anything we are interested in, and we can learn by looking for related teaching videos, which not only deepens our learning content but also improves our skill strengthening.

PSO is an evolutionary computing technology based on swarm intelligence, which solves the global optimal solution of iterative optimization problems. It finds the global optimum by following the optimal value currently searched. This algorithm has attracted the attention of academia due to its advantages such as easy implementation, high accuracy, and fast convergence and has demonstrated its superiority in solving practical problems. The algorithm principle is simple. Compared with genetic algorithms, there are no complex functions such as hybridization and mutation. They are just particles looking for the best particles in the solution space. Compared with other evolutionary algorithms, they have the advantage of being easy to apply and do not require complex models to solve optimization problems [18]. Since the field of evolutionary computing was proposed. As shown in Table 1, according to the number of commonly used search libraries, it can be seen that a large number of libraries and research results have emerged in a short period of more than ten years.

The particle swarm algorithm was first proposed by two American scientists based on the process of flocking birds to find the best foraging area. As an intelligent algorithm, PSO simulates the process of optimal decision-making. Similar to the human decision-making process, before you make a choice, will you be affected by your own experience (local optimal) and the experience of people around you (global optimal)? In the same way, the initial position of each bird is random during the foraging process of a group of birds. Of course, it is not known where the best foraging point is, and the flight direction of each bird is also random. It can be considered that in the early stage of foraging, the trajectory of the bird flock is chaotic. Over time, the birds in random positions learn from each other and share foraging information in the flock, combining his own experience and the information sent by his companions in the process of foraging to estimate the value of the food at the current location. Based on this search method, particle swarm optimization (PSO) came into being.

When the wireless signal propagates in free space and has an unobstructed straight path, the following formula can be used to calculate the distance $R$ between the transmitting node and the receiving node after the measurement data is obtained.

$$
p(r)=\frac{p_{k} G_{K} \beta^{2}}{(4 \pi)^{2} r^{2}} .
$$




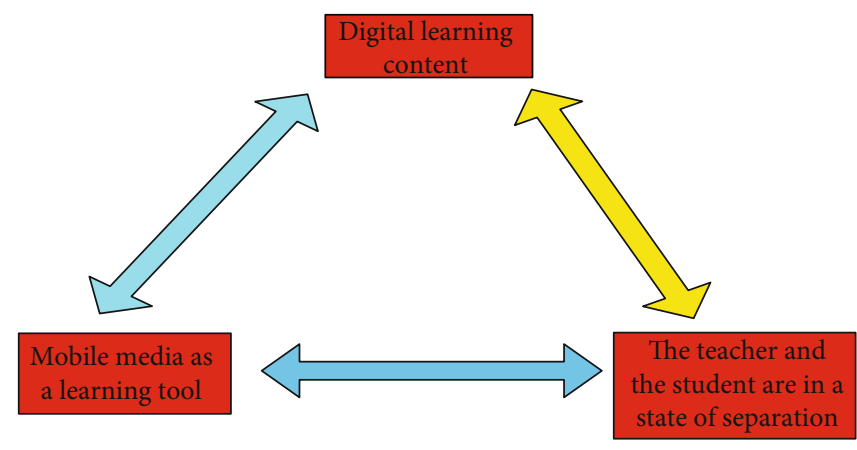

FIGURE 4: Judgment criteria for mobile learning.

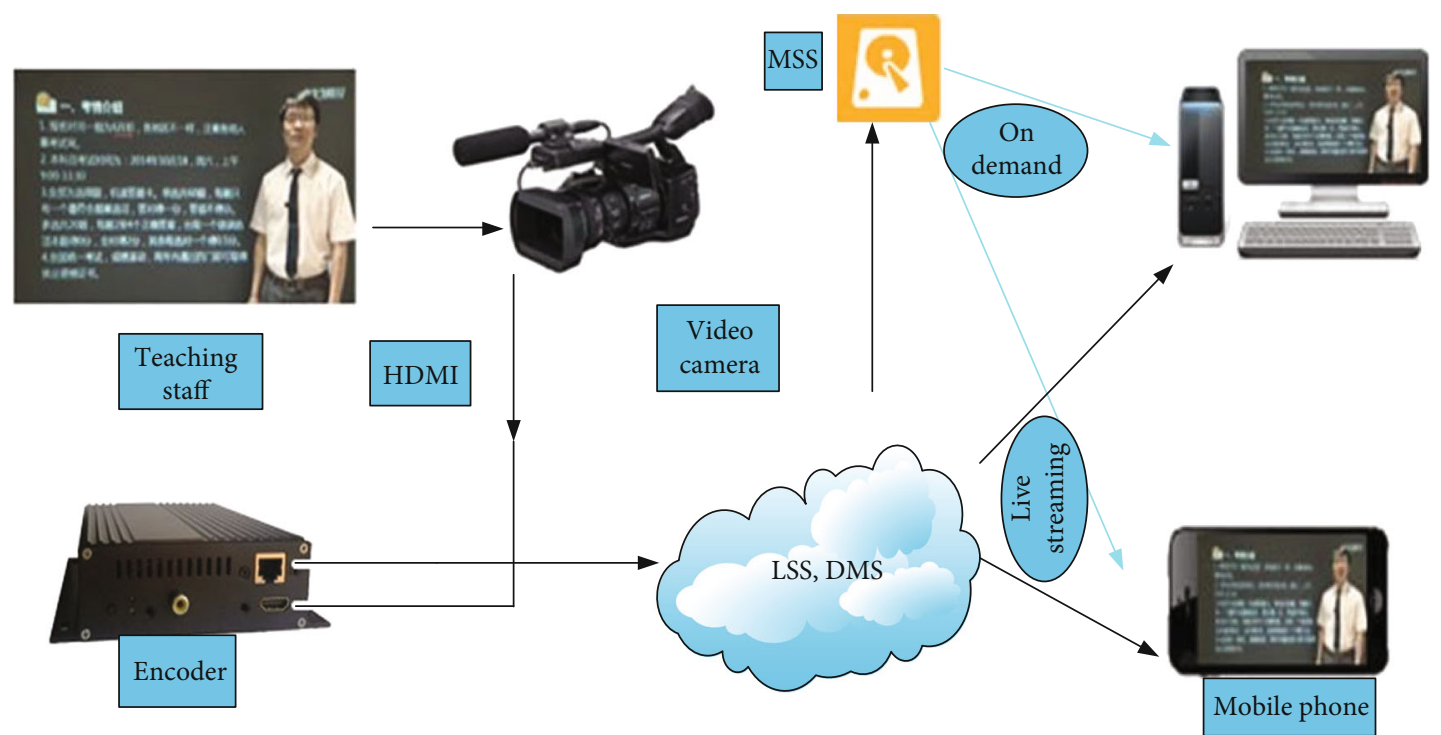

FIgURE 5: Online education live broadcast.

TABLE 1: Data statistics of particle swarm optimization algorithm in the literature library.

\begin{tabular}{lcc}
\hline Publication year & Records & Percentage \\
\hline 2008 & 300 & $3.20 \%$ \\
2009 & 400 & $6.20 \%$ \\
2010 & 445 & $7.10 \%$ \\
2011 & 523 & $7.50 \%$ \\
2012 & 625 & $8.60 \%$ \\
2013 & 800 & $9 \%$ \\
2014 & 654 & $9.50 \%$ \\
2015 & 911 & $10.23 \%$ \\
2016 & 240 & $15.66 \%$ \\
2017 & 1318 & $18.66 \%$ \\
2018 & 1097 & $19.60 \%$ \\
\hline
\end{tabular}

For the description of some parameters in the above formula, $P_{K}$ is the power of the transmitter, $p_{r}$ is the received power at the distance $r, r$ is the distance, $\beta$ is the wavelength, and the units are all meters; $G_{\mathrm{t}}$ and $G_{r}$ are the transmitting antenna and the receiving antenna, respectively. With this, we can calculate the distance between the transmitting point and the receiving point through a certain calculation.

The PSO algorithm has been widely used but there are also many problems. In order to solve these problems and in order to strengthen the search ability and convergence of PSO, some predecessors have introduced $\omega$ in the formula. The research shows that when $\omega$ is small, it narrows the network search range of the algorithm, and the powerful local search ability can improve the search accuracy. Balancing search accuracy and convergence speed is the focus of particle swarm optimization. The types of linearly decreasing inertia weights are

$$
v=v_{\max }-\frac{v_{\max }-v_{\min }}{t_{\max }} \times t .
$$

In the above formula, $v_{\max }$ is the maximum value of inertia weight, $v_{\min }$ is the minimum value of inertia weight, $t_{\max }$ is the maximum number of iterations, and $t$ is the current number of iterations. The linear inertia weight is globally optimized in the early stage of the particle swarm, and after finding the approximate position of the optimal solution, a local search is performed, which effectively improves the 
convergence speed and accuracy at the same time [19]. However, when the linearly decreasing weight solves complex problems, it is easy to ignore the population characteristics, the convergence speed slows down, and the optimal solution cannot be found, and it is easy to fall into the local optimum. Therefore, in order to balance the global search and the local search and solve the local optimal problem, this paper proposes a nonlinear adaptive inertia weight formula:

$$
v(k)= \begin{cases}v_{\max }-\frac{v_{\max }-(1 / 2) v_{\min }}{(1 / 2) t_{\max }} \cdot k, & k \leq \frac{1}{2} t_{\max }, \\ \left(\frac{1}{2} v_{\max }-v_{\min }\right) \cdot e^{-\left(k-(1 / 2) t_{\max }\right)}+v_{\min }, & k>\frac{1}{2} t_{\max } .\end{cases}
$$

In the above formula, $v_{\max }$ is the maximum value of inertia weight, $v_{\min }$ is the minimum value of inertia weight, and $t_{\max }$ is the maximum number of iterations, which is the current number of iterations. In the algorithm search process, the inertia weight of the particle swarm decreases linearly in the early stage $\left(k \leq(1 / 2) t_{\max }\right)$, and the particle swarm can often find the approximate position of the optimal solution quickly, which can prevent the particle swarm from falling into the local optimum in the early stage; in the late stage of the particle swarm $\left(k>(1 / 2) t_{\max }\right)$, the inertia weight decreases exponentially, which improves the search accuracy of the particle swarm to find the optimal solution more accurately. The improved inertia weight can adaptively change nonlinearly during the optimization process of the particle swarm. The high-speed optimization of the particle swarm in the early stage improves the convergence speed and at the same time improves the search accuracy of the network in the later stage and avoids the particle swarm from falling into the local optimum.

In the process of algorithm optimization, the initial stage should focus on global optimization, focusing on selflearning ability; in the later stage, the particle optimization range should be narrowed to quickly approach the global optimal, focusing on social learning ability [20]. The value of the learning factor $c_{1}$ first becomes larger and then smaller, and the value of the learning factor $c_{2}$ first becomes smaller and then larger. Based on this improved method to search for the global optimum of the network, improve the convergence speed of the algorithm, prevent the algorithm from falling into local freedom, and use the cosine algorithm to improve the learning factor.

$$
\begin{aligned}
& c_{1}=\left(j_{1}-j_{2}\right) * \cos \left(\frac{\pi}{2}\left(\frac{t}{t_{\max }}\right)\right)+j_{2}, \\
& c_{2}=\left(j_{3}-j_{4}\right) * \cos \left(\frac{\pi}{2}\left(\frac{t}{t_{\max }}\right)\right)+j_{4} .
\end{aligned}
$$

In the above formula, $j_{1}, j_{2}, j_{3}$, and $j_{4}$ are the starting value and final value of $c_{1}$ and $c_{2}$, respectively. After many experiments, we get $j_{1}=3.5, j_{2}=0.5, j_{3}=2.5$, and $j_{4}=0.5$. When the algorithm is used, do it right.
2.4. Optimal Number of Cluster Heads. In the algorithm performance comparison of wireless sensor networks, clustering can effectively reduce network energy consumption and improve performance. Assuming that there are $g$ nodes in an $m \times m$ network, the number of cluster heads is $b$, so the probability of a node being elected as a cluster head is $g / b$, the number of nodes distributed in each cluster is $(g / b)-1$, and the number of nodes in each cluster is JJ. In the calculation, the energy consumed by the base station during signal transmission is

$$
E=\left(\frac{g}{b}-1\right) \times e_{\mathrm{el}} \times l+\frac{g}{b} \times E_{\mathrm{DA}} \times l+\left(E_{\mathrm{el}} \times l+\eta_{\mathrm{am}} \times r_{\mathrm{to}}^{4} \times l\right) .
$$

In the above formula, $r_{\text {to }}$ is the distance from the cluster head to the base station. When a node transmits data to the cluster head, it consumes a certain amount of energy.

$$
E_{\mathrm{me}}=l \times E_{\mathrm{el}}+l \times \eta_{\mathrm{f}} \times \mathrm{r}_{\mathrm{to}}^{2}
$$

In the above formula, $r_{\text {to }}$ is the distance from the node to the cluster head. Assuming that each node is $p(x, y)$, its expectation is

$E\left[\mathrm{r}_{\mathrm{to}}^{2}\right]=\iint\left(x^{2}+y^{2}\right) p(x, y) d x d y=\iint\left(x^{2}+y^{2}\right) \frac{b}{m^{2}} d x d y=\frac{m^{2}}{2 b \pi}$.

The total energy consumed is

$E_{\mathrm{to}}=b E_{\mathrm{CH}}=l \times\left[(2 g-b) \times E_{\mathrm{el}}+\frac{m^{2}}{2 b \pi}(\mathrm{g}-\mathrm{b}) \eta_{\mathrm{f}}+e_{\mathrm{da}} g+\mathrm{b} \eta_{\mathrm{am}} \mathrm{r}_{\mathrm{to}}^{4}\right]$.

Solve the first derivative of $E$ with respect to $b$, and make it equal to zero, so that we can get

$$
\eta_{\mathrm{am}} \times r_{\mathrm{to}}^{4}-\eta_{\mathrm{f}} \times \frac{g}{2 \pi} \times \frac{m^{2}}{b^{2}}=0
$$

Solve the above equation to get

$$
b=\sqrt{\frac{\eta_{\mathrm{f}}}{\eta_{\mathrm{am}}}} \cdot \sqrt{\frac{g}{2 \pi}} \cdot \frac{m}{r_{\mathrm{to}}^{2}} .
$$

The decision factor $f_{1}$ is based on the network energy consumption, the decision factor $f_{2}$ is based on the remaining energy of the cluster head, the decision factor $f_{3}$ is based on the distance between the cluster head and the nodes in the cluster, and the decision factor $f_{4}$ is based on the distance between the cluster head and the base station. According to the previous energy consumption model, we can get the total energy consumption of network communication as 


$$
E_{\mathrm{to}}=\sum_{g=1}^{g} l \times\left[\left(2\left|c_{b}\right|-1\right) \times E_{\mathrm{el}}+r_{\mathrm{to}}^{2}\left(\left|c_{b}\right|-1\right) \eta_{\mathrm{f}}+\left|c_{b}\right| e_{\mathrm{da}}+\eta_{\mathrm{am}} r_{\mathrm{to}}^{4}\right] .
$$

Then, we can get the power consumption judgment factor of the network node as

$f_{1}=\frac{1}{g} \sum_{g=1}^{g} l \times\left[\left(2\left|c_{b}\right|-1\right) \times E_{\mathrm{el}}+r_{\mathrm{to}}^{2}\left(\left|c_{b}\right|-1\right) \eta_{\mathrm{f}}+\left|c_{b}\right| e_{\mathrm{da}}+\eta_{\mathrm{am}} r_{\mathrm{to}}^{4}\right]$.

In the definition of decision factor $f_{2}$, first sum up the remaining energy of all particle nodes in the algorithm, and divide it by the energy of the cluster head. The smaller the value obtained, the greater and better the possibility of being selected as the cluster head.

$$
f_{2}=\frac{\sum_{i=1}^{g} E}{\sum_{b=1}^{b} e(c h)} .
$$

The definition of decision factor $f_{3}$ is to calculate the maximum average Euclidean distance between a common node and its cluster head according to the node position. The smaller the value, the better the compactness.

$$
f_{3}=\sum_{i=1}^{\mathrm{g}} \sum_{b=1}^{b} \frac{d\left(n_{i}, \mathrm{ch}\right)}{\left|C_{P, K}\right|} .
$$

$f_{4}$ is the minimum distance between the node and the base station. The smaller the distance from the base station, the lower the communication energy consumption.

$$
f_{4}=\min \left\{d\left(\mathrm{ch}_{i}, \mathrm{bs}\right)\right\} .
$$

In the above formula, $d\left(\mathrm{ch}_{i}, \mathrm{bs}\right)$ is the distance from the $i$ th cluster head to the base station. Based on the above four decision factors, the decision function constructed in this article is

$$
\text { cost }=\beta f_{1}+\alpha f_{2}+\chi f_{3}+\gamma f_{4} .
$$

$f_{1}, f_{2}, f_{3}$, and $f_{4}$ are the decision factors of this algorithm when selecting cluster heads. The specific physical meanings of the four factors are as described above. The parameters $\alpha, \beta$ ,$\lambda$, and $\eta$, respectively, represent the weight of each decision factor in the fitness function, $\alpha \beta \gamma \eta \varepsilon[0,1], \alpha+\beta+\lambda+\eta=1$, and the weights of the four decision-making factors can be adjusted according to actual needs [21]. According to the definition of fitness function, a relatively small fitness function value can ensure the selection of the optimal cluster head. The smaller the fitness function of the particle, the less the energy consumption of the network, the more the remaining energy of the cluster head node, the better the compactness within the cluster, and the better the energy consumption of the cluster head communication. Therefore, the goal of improving the particle swarm algorithm is to select the node with the smallest fitness function value as the cluster head, optimize the selection of the cluster head, and reduce network energy consumption.

In the initial stage of the algorithm, each node randomly generates a random number $\mu, 0<\mu<1$, and sets the thresholdTfor electing cluster heads, $\mu<T$; the node is elected as the candidate cluster head. In the improvement of the threshold $T$, the influence of the global network node is taken into consideration, and comprehensive consideration is given to node energy, density, and distance from the base station which are three factors.

$$
\begin{gathered}
T(n)\left\{\begin{array}{cc}
\frac{p}{1-p(d * m i(1 / p))} \cdot \frac{e_{i}}{e_{0}} \cdot \frac{d_{i}-d_{\min }}{d_{\text {max }}-d_{\min }}, & n \in g, \\
0, & n \notin g,
\end{array}\right. \\
Q_{i}=\frac{\mathrm{ne}_{i}(i)_{\mathrm{ali}}}{\mathrm{net}_{\text {ali }}} \\
x_{i j}(h+1)=x_{i j}(h)+v_{i j}(h+1) .
\end{gathered}
$$

In the above formula, there is a probability that a network node is elected as a cluster head in normal times, where $p$ is the probability that a network node is elected as a cluster head, $r$ is the number of running rounds of the network at this time, and $G$ is the set of nodes that are elected as cluster heads in the most recent $1 / p$ round. $E_{0}$ and $E_{\mathrm{i}}$ are the initial energy and current energy of the node, respectively; $d_{\max }$ and $d_{\min }$ are the longest and shortest distances between the network node and the sink node, $d$ is the distance between the node and the base station, and $Q_{i}$ is the node density. $\mathrm{Ne}_{i}$ is the number of neighbor nodes of node $i$, and Net is the number of surviving nodes in the network. The candidate cluster head set is constructed through the determination of the candidate cluster heads. The candidate cluster heads only unilaterally determine the energy and location information of the nodes. The energy consumption of the nodes and the global network information are not considered, and the election of the algorithm cannot be optimized. There is a need to optimize the election of the network candidate cluster head [22].

Test experiments and practice have proved that the quantum particle swarm optimization algorithm shows good solving characteristics. The improved algorithm adds particles with quantum behavior, breaking the limitations of particle motion speed and position, so the particle swarm optimization algorithm with quantum behavior shows. In addition to the excellent features that the particle swarm optimization algorithm cannot match, many aspects have been improved. In order to meet this exponential increase in data throughput, heterogeneous networks will achieve better performance in terms of increasing network capacity, increasing coverage, and improving spectrum efficiency. At the same time, the unprecedented rapid development of wireless communication technology is shown in Figure 6. 


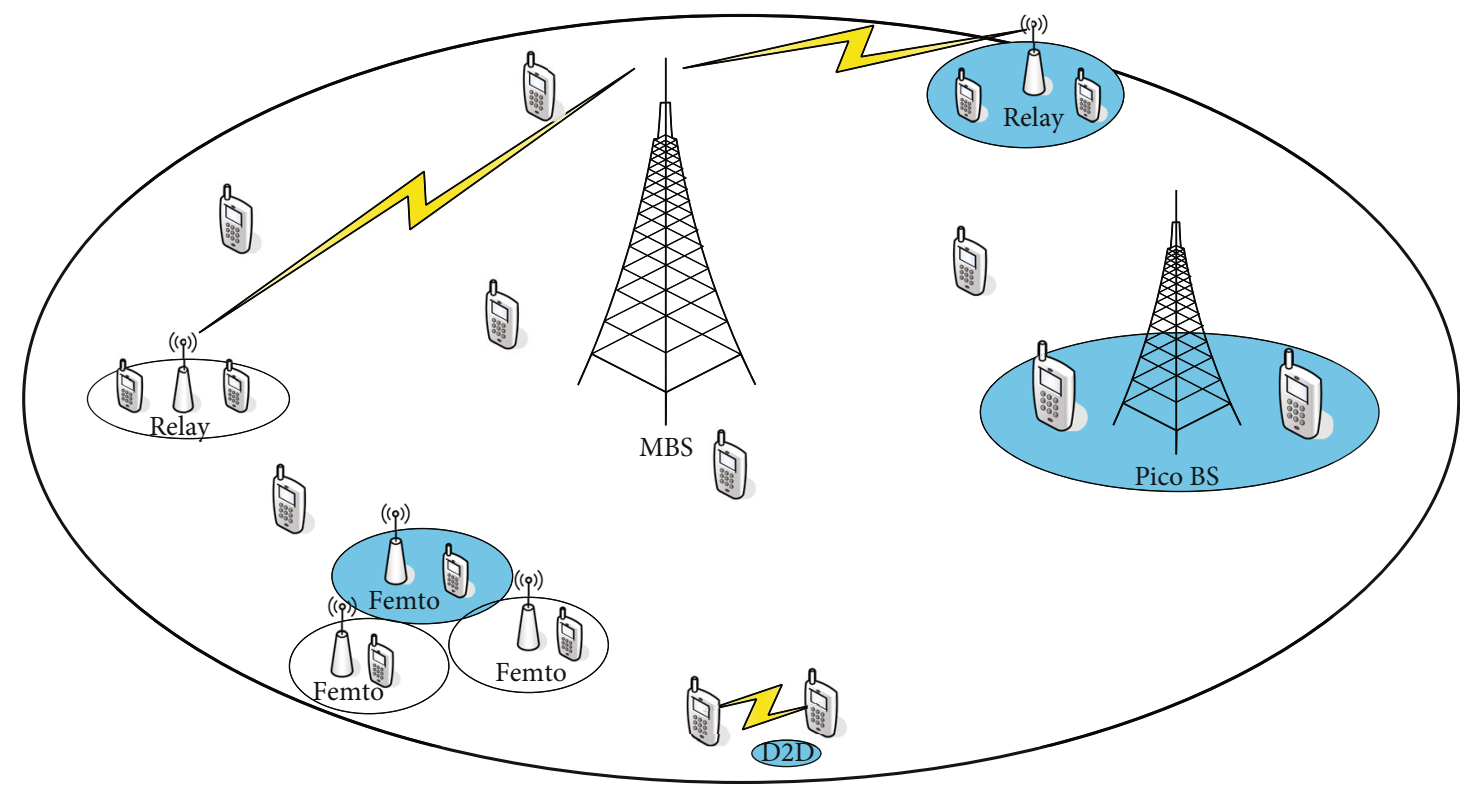

Figure 6: Multilayer heterogeneous network architecture model.

\section{Contrast Experimental Design}

3.1. Experimental Environment. In order to test the wireless channel allocation performance of the combined intelligent algorithm, Matlab 2016 was selected as the experimental platform. The parameters of the wireless network and the combined intelligent algorithm are defined as follows: the number of nodes is 40 , the communication radius is $100 \mathrm{~m}$, the interference radius is $30 \mathrm{~m}$, the total number of available channels is 24 , the population is 100 for the genetic algorithm, the maximum evolutionary number of the genetic algorithm is 1000 , the population number of the swarm optimization algorithm is 40 , and the maximum number of iterations of the particle swarm optimization algorithm is 500, $\delta=0.95, C_{1}=1.95$, and $C_{2}=1.5$. Select the wireless channel allocation method in literature [9] and bibliography [13] for benchmark testing.

For genetic algorithm, it is difficult to obtain the global optimal wireless network channel allocation plan when solving the wireless network channel allocation problem, and the particle swarm optimization algorithm for the wireless network channel allocation problem solves the slower problem, and the combination of them is improved. The throughput of the wireless network, the network delay, and the packet loss rate of data transmission are smaller, and the communication performance of the wireless network is significantly improved.

The wireless network channel allocation process is mainly divided into three stages: data collection, wireless network channel allocation method design, and wireless network channel allocation implementation. Among them, data collection is used to collect wireless network-related parameters and working environment data to provide data for the wireless network channel allocation method; and the wireless network channel allocation method design mainly deter-

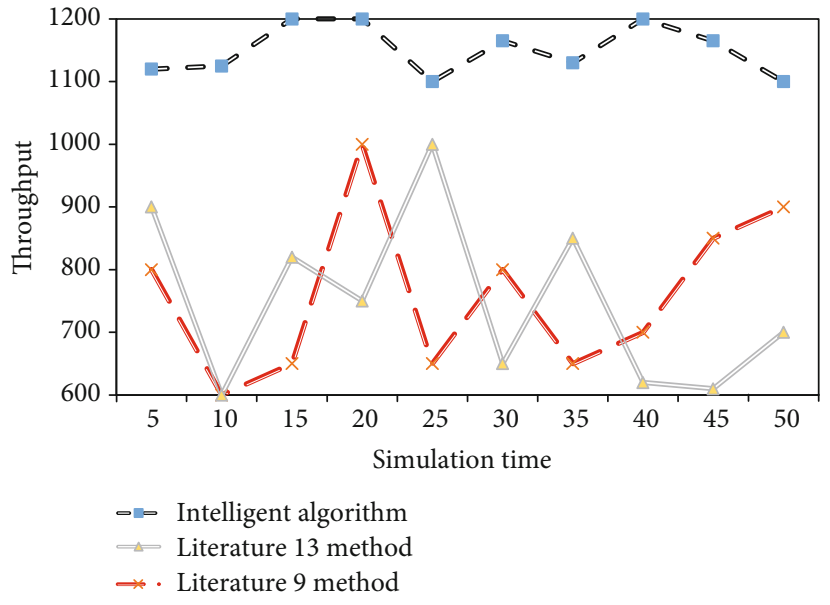

Figure 7: The change curve of the throughput of the wireless network communication system.

mines the optimal wireless network channel allocation plan and finally obtains the wireless network channel. The allocation scheme is applied in the specific wireless network communication process, so the design of the wireless network channel allocation method is the most critical.

3.2. Throughput Comparison of Wireless Network Communication Systems. Throughput mainly describes the amount of data received by the wireless network communication system in a unit of time. The performance change curve of the wireless network communication system under different simulation times and three wireless network channel allocation methods is shown in Figure 7 [23]. It shows that the wireless channel allocation method used in this paper is relatively stable to the changes in the performance of the wireless communication system; it shows that the 


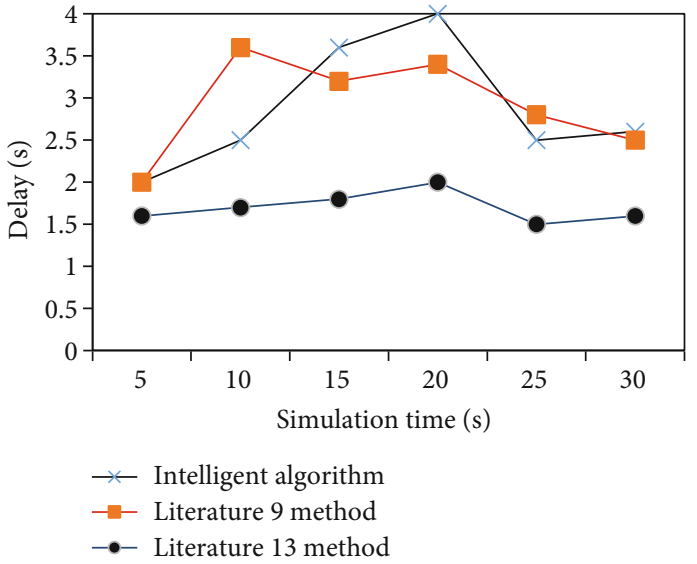

(a)

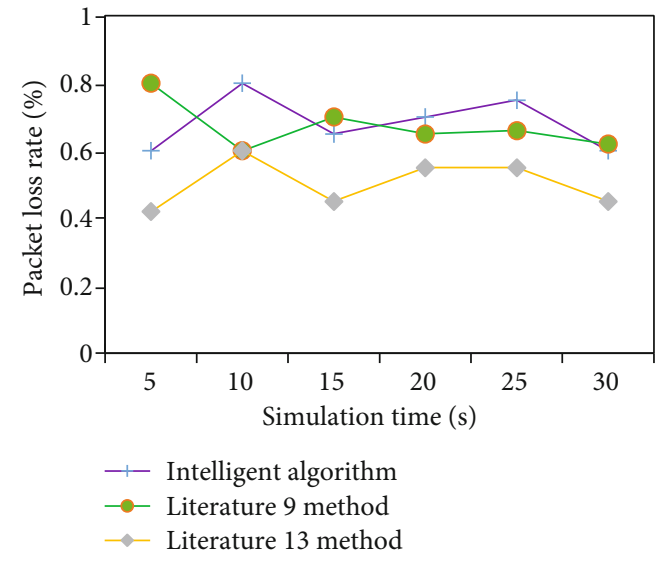

(b)

Figure 8: (a) Transmission delay variation curve of wireless network communication system. (b) The packet loss rate of the wireless network communication system changes with time.

performance of the wireless network communication system is unstable. At the same time, the network communication system of the wireless channel allocation method in this paper is significantly higher than that of the literature [9] and literature (the wireless communication system of the method in 13) shows that the wireless network distribution system implemented by this method makes it more meaningful to make full use of the wireless network and increase the utilization rate of the wireless network.

3.3. Problems in the Process of Wireless Network Transmission. In the same experimental environment, the transmission delay curves of the wireless network communication system with different simulation times and three wireless channel allocation methods are shown in Figure 8. Point (a) shows that the transmission delay of this method of wireless communication network is significantly shorter than that of literature [9] and literature [13], which is almost 5\%. Accelerated data transmission the wireless network communication system is fast, can be applied to timesensitive occasions, and has high practical application value. It can be seen from point (b) that the packet loss rate of the wireless communication network in reference [9] and reference [13] is generally high, indicating that the wireless network is subject to great interference, and the probability of network congestion is high and wireless channel allocation is unreasonable. This wireless network communication method has a low packet loss rate and fewer wireless network data transmission errors, which is nearly $10 \%$ lower than the other two methods. This improves the wireless data transmission capacity of the communication network.

Through reading other documents, it is found that the packet loss rate of their wireless communication network is generally high, but this wireless network communication method in this article has a low packet loss rate and fewer wireless network data transmission errors, which is more energy-efficient and faster than the market. By about 15\%, the wireless data transmission capacity of the communication network has been improved.

\section{Discussion of Experimental Results}

4.1. Energy Consumption during Signal Transmission. As shown in Figure 9, when the distance between the transmitting end and the receiving end of the user changes from $10 \mathrm{~m}$ to $100 \mathrm{~m}$, the total energy efficiency of D2D users under both methods increases with the increase of the distance between TX and RX in D2D users and reduces. This is because as the distance between TX and RX increases, the path loss increases, resulting in greater power consumption, which will have a serious impact on the energy effect of D2D communication. At the same time, we can see that the RR algorithm proposed in this chapter is better than the TM algorithm in terms of energy efficiency under the QoS requirements of the two cell users. When the minimum rate requirement for cell users increases, the gap between the RR algorithm and the TM algorithm in terms of D2D communication energy efficiency will be reduced. This is because when Rc is large, in order to avoid crosslayer interference, the transmission power of D2D users will be strictly limited [24]. Through the RR algorithm with intelligent algorithm, we found that they can reduce energy consumption by nearly $10 \%$ without the help of intelligent algorithm in the process of wireless network propagation, which is above the same distance., We also found in the TM algorithm that with the blessing of intelligent algorithms, only the energy consumption has been reduced by $7 \%[25,26]$.

4.2. The Purpose of Using Cloud Education by Students. The source of the experimental data is a questionnaire survey of 550 issued for each class of a certain university. The questionnaire stars used are drawn based on the results of the questionnaire survey we have obtained. In order to understand how students use various electronic device cloud education platforms for learning at different levels, because we felt that the data obtained was not true enough, we adopted offline and distributed some questionnaires in the cafeterias of various schools in the university city for corresponding recovery. The recovery rate reached $100 \%$. A total of 550 questionnaires were collected, and 480 valid questionnaires 


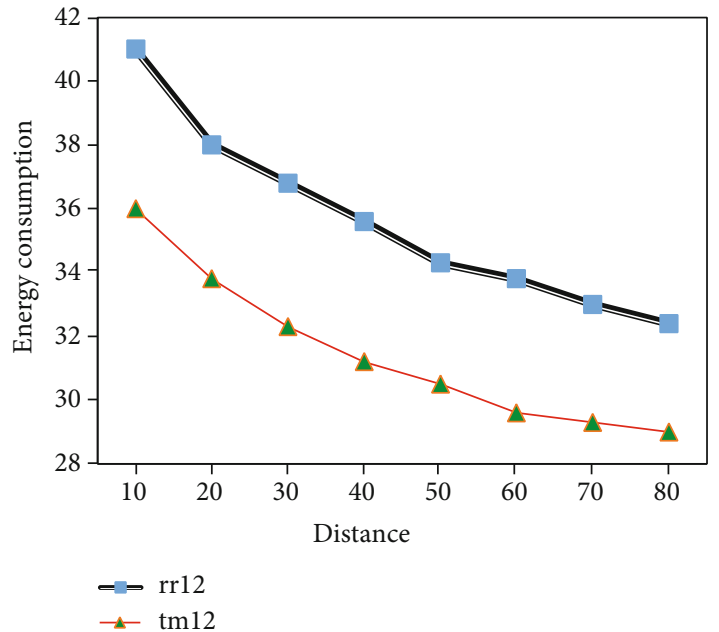

(a)

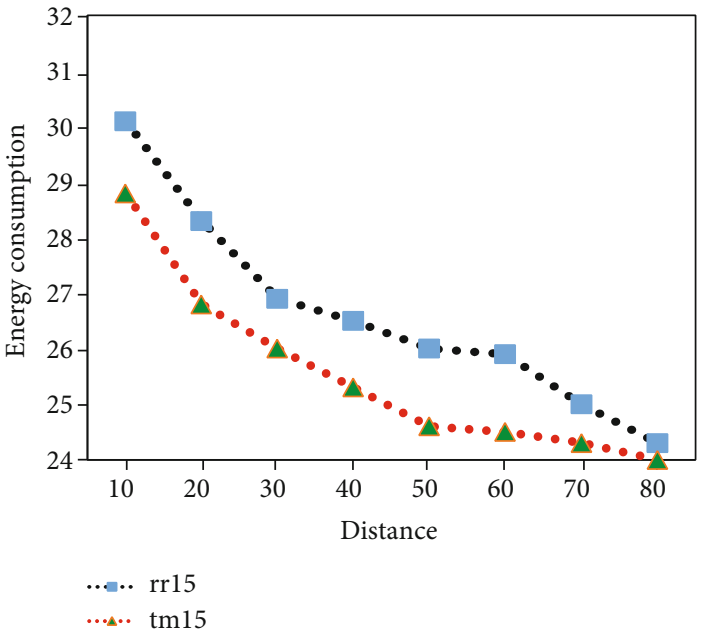

(b)

FIGURE 9: (a) The relationship between propagation energy consumption rr12 and tm12 and distance. (b) The relationship between propagation energy consumption rr15 and tm15 and distance.

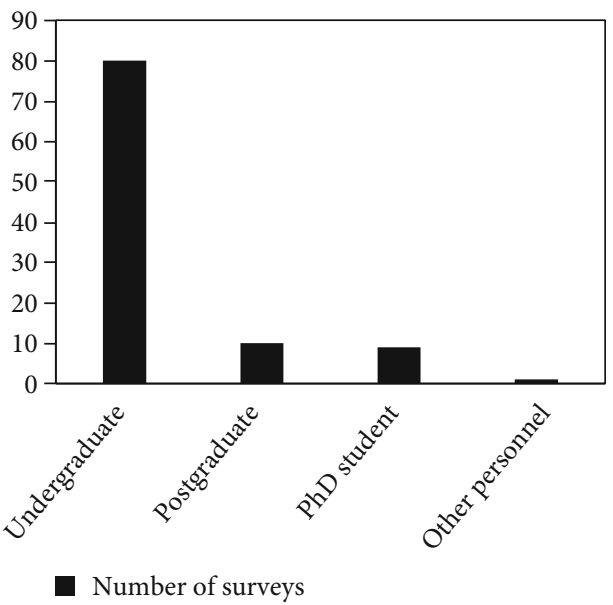

(a)

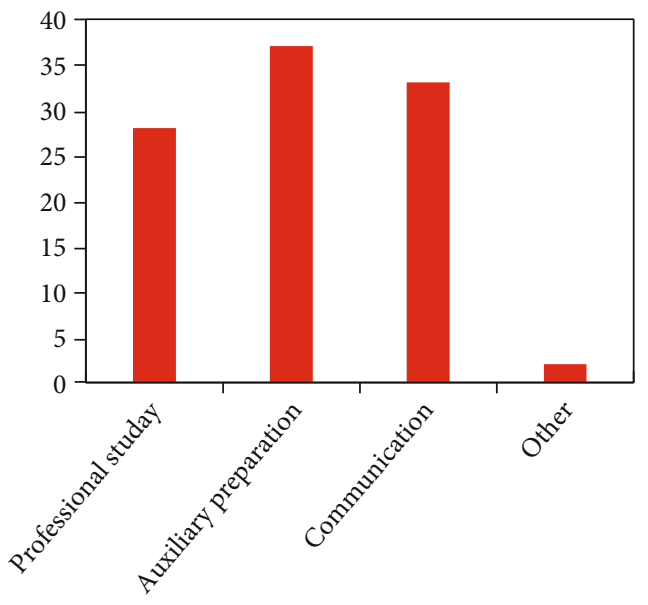

(b)

Figure 10: (a) Educational qualifications of investigators. (b) Purpose of participating in mobile learning.

were collected. The effective rate is $87.28 \%$. According to the returned questionnaire, it can be clearly seen from Figure 10 (a) that $80 \%$ of the survey subjects are undergraduates (384), 10\% are master students (48), and $9 \%$ are doctoral students (48 people); $1 \%$ is other personnel ( 5 people); $98 \%$ of the students own laptops, among which the holding rate of notebooks for masters and doctoral students is $100 \%$, and the holding rate of iPad reaches $95 \%$. We can see from Figure 10(b) that the purpose of students participating in mobile learning is not consistent, basically considering individual needs (note: this data excludes nonlearning purpose behavior), but mainly concentrated in three aspects, $28 \%$ of students. It is believed that mobile learning can acquire new knowledge and assist professional learning in the classroom environment. $37 \%$ of students use mobile learning to assist in exams, as a means of preparing for exams, especially for time users such as review of final exams and college English test bands 4 and 6 . Moreover, 33\% of the students used mobile learning for teacher-student exchanges and discussions, $2 \%$ of the students used mobile learning for other purposes such as entertainment and emotional communication [27], as shown in Figure 10.

\section{Conclusions}

The learning that cloud education will bring to us will be when we are at home, turn on the computer or mobile phone, and wait for any mobile terminal device that can access the Internet, and timely check the missing and fill vacancies of what we learned on the day, summarize, and review; on the bus, complete the day's assignments which are automatically submitted; while traveling or doing other 
things while listening to teaching videos, or learning the latest information, we will be in the cloud campus, the smart campus of the cloud classroom. It can help us enrich our knowledge through cloud education.

The American Annual Education Technology Report points out that globalization, networking, and digitization have brought about a big explosion of knowledge, and people's learning time and learning space are extending infinitely. The study time broke through the fixed hours of study or work and began to expand to sleep and lifetime. The learning space also began to break through the boundaries of the school and gradually expanded to the society. Future education must be a knowledge dissemination mode that meets the needs of all kinds of learners and supports multiple learning methods. Use advanced information technology to build a modern education and teaching environment and realize the integration of people and campus, people and information system, and campus entity and information system, so as to improve teaching methods, promote scientific research, improve management efficiency, and improve service level. As new technologies, new ideas, and new applications emerge, cloud education has many advantages. However, we should also see that before any new thing matures, there will always be more or less defects, and cloud education is no exception. Mobile learning is convenient and fast, but it is difficult for learners to concentrate for a long time. We can split the learning content into small knowledge points to suit learners to quickly learn in a short time. In addition, rich resources are a major advantage of cloud education. However, massive learning resources can make learners lose or feel at a loss. Then, when we build a network resource library, we must fully consider the needs of users and use the resources most appropriately. The way of organization is presented to students, allowing them to truly experience the convenience and speed brought by "cloud computing" technology to resource retrieval. How to make cloud education play its true value and advantages; realize anytime, anywhere; and maximize the benefits, to make up for the lack of resources and support services for extracurricular learning, we need to further explore. The development of cloud education is the general trend. It is believed that cloud education will play a huge role in promoting the development of mobile learning and bring disruptive changes to mobile learning. Perhaps, in the near future, the learning we will see will be as follows: when the learner is at home, turning on the computer or mobile phone and waiting for any mobile terminal device that can access the Internet and timely checking the missing and filling vacancies of the content of the day, summing up, and reviewing; in the bus and in the car, complete the day's homework and automatically submit it; while traveling or doing other things, listen to the instructional video, or learn the latest information; we will be in the cloud campus, the smart campus of the cloud classroom. I believe that we will become more and more intelligent in the future.

\section{Data Availability}

No data were used to support this study.

\section{Conflicts of Interest}

The authors state that this article has no conflict of interest.

\section{Acknowledgments}

This work was supported by the project of higher education teaching reform of Chongqing Municipal Education Commission in 2018: Research on the Development, Construction and Application of Series of Online Courses for Teacher Education (183040). This work was supported by Education Department of Jiangxi Province Science and Technology Research Project-Indoor Positioning Research for Educational Robots (GJJ202119).

\section{References}

[1] Y. Wang, M. Zhang, and W. Shu, "An emerging intelligent optimization algorithm based on trust sensing model for wireless sensor networks," Eurasip Journal on Wireless Communications \& Networking, vol. 2018, no. 1, pp. 145-148, 2018.

[2] C. Lin, G. Wu, T. Qiu, and J. Deng, "A low-cost node capture attack algorithm for wireless sensor networks," International Journal of Communication Systems, vol. 29, no. 7, pp. 12511268, 2016.

[3] C. Tang, Q. Tan, and Y. Han, "An energy harvesting aware routing algorithm for hierarchical clustering wireless sensor networks," KSII Transactions on Internet and Information Systems(TIIS), vol. 2, pp. 504-521, 2016.

[4] F.-I.-A. Muhammad, "Intelligent energy efficient localization using variable range beacons in industrial wireless sensor networks," IEEE Transactions on Industrial Informatics, vol. 12, no. 6, pp. 2206-2216, 2016.

[5] P. Sengottuvelan and N. Prasath, "BAFSA: breeding artificial fish swarm algorithm for optimal cluster head selection in wireless sensor networks," Wireless Personal Communications, vol. 94, no. 4, 2017.

[6] Y. Xu, "Simulation of optimal selection algorithm for wireless sensor cluster head node Bayesian statistical network," Journal of Intelligent and Fuzzy Systems, vol. 1, pp. 1-9, 2021.

[7] S. A. Kati and M. A. Khan, "Challenges and issues of Elearning using education cloud - a review in context of COVID-19 pandemic," International Journal of Computer Applications Technology and Research, vol. 10, no. 5, pp. 106-110, 2021.

[8] S. Li, B. Duo, X. Yuan, Y.-C. Liang, and M. Di Renzo, "Reconfigurable intelligent surface assisted UAV communication: joint trajectory design and passive beamforming," IEEE Wireless Communications Letters, vol. 9, no. 5, pp. 716-720, 2020.

[9] X. Yang, D. Yang, and S. Aue, "Intelligent encryption algorithm for network communication parallel data of information release terminal," Journal of Intelligent \& Fuzzy Systems, vol. 35, no. 4, pp. 4245-4255, 2018.

[10] S. Sasirekha and S. Swamynathan, "Cluster-chain mobile agent routing algorithm for efficient data aggregation in wireless sensor network," Journal of Communications \& Networks, vol. 19, no. 4, pp. 392-401, 2017.

[11] K. Soga, S. Hara, M. Motoki, and S. Sasaki, "The landmark detection method using SURF and Q-learning for an autonomous mobile robot," Ieej Transactions on Electronics Information \& Systems, vol. 137, no. 9, pp. 1248-1257, 2017. 
[12] Y. Wang, J. Yue, Y. Dong, and Z. Hu, "Review on kernel based target tracking for autonomous driving," Journal of Information Processing, vol. 24, no. 1, pp. 49-63, 2016.

[13] X. T. Truong, T. D. Hong, and D. N. Cong, "An efficient navigation framework for autonomous mobile robots in dynamic environments using learning algorithms," Journal of Computer Science and Cybernetics, vol. 33, no. 2, pp. 107-118, 2018.

[14] T. Takebayashi, R. Miyagusuku, and K. Ozaki, “Development of magnetic-based navigation by constructing maps using machine learning for autonomous mobile robots in real environments," Sensors, vol. 21, no. 12, pp. 3972-3974, 2021.

[15] Q. Ailing, "A study on college English autonomous learning model based on ESP theory," Agro Food Industry Hi Tech, vol. 28, no. 1, pp. 904-907, 2017.

[16] S. Ghosh, A. Pal, S. Jaiswal, K. C. Santosh, N. Das, and M. Nasipuri, "SegFast-V2: semantic image segmentation with less parameters in deep learning for autonomous driving," International Journal of Machine Learning and Cybernetics, vol. 10, no. 11, pp. 3145-3154, 2019.

[17] J. Zhai and J. Bi, "Empirical research of college students' learning autonomy from the perspective of intelligent mobile learning platform," Boletin Tecnico/Technical Bulletin, vol. 55, no. 8 , pp. 266-273, 2017.

[18] H. M. Sabi, F.-M. E. Uzoka, K. Langmia, and F. N. Njeh, "Conceptualizing a model for adoption of cloud computing in education," International Journal of Information Management, vol. 36, no. 2, pp. 183-191, 2016.

[19] I. W. Park and J. Han, "Teachers' views on the use of robots and cloud services in education for sustainable development," Cluster Computing, vol. 19, no. 2, pp. 987-999, 2016.

[20] F. Karim and G. Rampersad, "Cloud computing in education in developing countries," Computer \& Information Science, vol. 10, no. 2, pp. 87-96, 2017.

[21] H. Lin, "The construction of mobile education in cloud computing," Procedia Computer Science, vol. 183, no. 8, pp. 14$17,2021$.

[22] G. Hu, Y. Yang, and L. Li, "Education technology cloud platform framework establishment and security," Journal of Computer and Communications, vol. 4, no. 7, pp. 7-14, 2016.

[23] M. A. Alam and A. Saiyeda, "A cloud based solution for smart education," International Journal of Smart Education and Urban Society, vol. 11, no. 2, pp. 28-37, 2020.

[24] A. Dadamuxamedov, X. Mavlyuda, and J. Turdali, "Cloud technologies in Islamic education institutions," ACADEMICIA An International Multidisciplinary Research Journal, vol. 10, no. 8, pp. 542-546, 2020.

[25] Z. Lv, "The security of Internet of drones," Computer Communications, vol. 148, pp. 208-214, 2019.

[26] A. Zl, A. Dc, A. Rl, and B. Aa, "Artificial intelligence for securing industrial-based cyber-physical systems," Future Generation Computer Systems, vol. 117, pp. 291-298, 2021.

[27] G. Yan and R. Sha, "Autonomous learning of foreign language based on facial emotion recognition and cloud computing," Journal of Intelligent and Fuzzy Systems, vol. 11, pp. 1-12, 2020. 